\title{
Whipple Procedure
}

National Cancer Institute

\section{Source}

National Cancer Institute. Whipple Procedure. NCI Thesaurus. Code C15356.

Surgical removal of part of the pancreas, duodenum, and distal part of the bile duct.

Sometimes, the lower portion of the stomach and the gallbladder are also removed. This procedure is performed for pancreatic, ampullary, duodenal and distal bile duct cancers. 